\title{
Performance of a Ge-microstrip imaging detector and polarimeter
}

\author{
U. Spillmann, ${ }^{1}$ H. Bräuning, ${ }^{1}$ S. Hess, ${ }^{1}$ H. Beyer, ${ }^{1}$ Th. Stöhlker, ${ }^{1}$ J.-Cl. Dousse, ${ }^{2}$ \\ D. Protic, ${ }^{3}$ and T. Krings ${ }^{3}$ \\ ${ }^{1}$ Gesellschaft für Schwerionenforschung mbH, Planckstr. 1, 64291 Darmstadt, Germany \\ ${ }^{2}$ Department of Physics, University of Fribourg, CH-1700 Fribourg, Switzerland \\ ${ }^{3}$ Forschungszentrum Jülich GmbH, Institut für Kernphysik, 52425 Jülich, Germany
}

\begin{abstract}
Using 98\% linearly polarized radiation at the European Synchrotron Radiation Facility in Grenoble, the performance of a prototype two-dimensional microstrip Ge(i) detector for x-ray imaging and as a Compton polarimeter has been evaluated. Using the energy and position sensitivity of the detector, the ability to obtain a complete reconstruction of the Compton event has been demonstrated. The modulation coefficient of the polarimeter is in good agreement with the theoretical limit of a perfect detector.
\end{abstract}

\section{INTRODUCTION}

In the recent years, state-of-the-art x-ray detectors have become available, which combine the good efficiency, energy, and time resolution of semiconductor detectors with submillimeter position resolution and a large detection area. Previously, a submillimeter position resolution in large area detectors was the domain of gaseous detectors or scintillators, while high energy resolutions require semiconductor detectors [i.e., $\mathrm{Si}(\mathrm{Li})$ or $\mathrm{Ge}(\mathrm{i})$ ]. Recent developments in the methods of photolithography and plasma etching now permit the production of two-dimensional Ge(i) microstrip detectors with a pitch in the order of a few hundred micrometers. Such detectors have a broad range of applications from medical imaging and applied science to basic research such as astrophysics ${ }^{1}$ and nuclear or atomic physics. ${ }^{2,3}$

In highly charged heavy ions, for example, x-ray spectroscopy is the major tool to study the structure and dynamics of simple atomic few-electron systems in high electric fields. ${ }^{3,4}$ Here the highest resolution is obtained with crystal spectrometers such as FOCAL (Ref. 5) for hard x-rays. These require large area $\mathrm{x}$-ray detectors for efficiency with submillimeter position resolution and very good linearity. The two-dimensional position sensitivity furthermore allows for the correction of the Doppler effect in fast-beam experiments. The additional energy and time resolution offered by the position sensitive semiconductor detectors has been proven to be essential for background suppression.

Besides their application in classical spectroscopy, such detectors will work as highly efficient Compton polarimeters. This opens a new field to study polarization of $\mathrm{x}$-rays from highly charged ions. ${ }^{6} \mathrm{~A}$ major part of the $\mathrm{x}$-ray experiments planned within the SPARC collaboration ${ }^{7}$ at the future FAIR facility ${ }^{8}$ will depend on such new detectors.

In this paper we report on a novel prototype $\mathrm{Ge}(\mathrm{i})$ detector built at IKP Jülich, ${ }^{9}$ which features a good energy and time resolution of semiconductor detectors together with a submillimeter position resolution. Designed for use with a crystal spectrometer, ${ }^{5,10}$ a position resolution of $250 \mu \mathrm{m}$ in the direction of the dispersion and $1167 \mu \mathrm{m}$ in the orthogo- nal direction was chosen. Using a well focused x-ray beam from the European Synchrotron Radiation Facility (ESRF), we evaluate here its detection characteristic such as its linearity in the position measurement. Employing the high degree of linear polarization of the $\mathrm{x}$-rays provided by the ESRF, we also show the detector's superb characteristics as a Compton polarimeter.

\section{DETECTOR}

For the detector a single crystal of high purity $n$-type germanium with a total area of $41 \times 70 \mathrm{~mm}^{2}$ and a thickness of $11 \mathrm{~mm}$ has been used. The front side consists of a boron implanted $p^{+}$contact. It is divided by plasma etching into 128 strips of $56 \mathrm{~mm}$ length and a pitch of $250 \mu \mathrm{m}$. The strips are separated by $28 \mu \mathrm{m}$ wide and $15 \mu \mathrm{m}$ deep grooves. The back side is an amorphous Ge contact, which has been segmented by plasma etching into 48 strips. These strips have a length of $32 \mathrm{~mm}$ with a pitch of $1167 \mu \mathrm{m}$ and are oriented perpendicular to the strips on the front side (see Fig. 1). The active area of $32 \times 56 \mathrm{~mm}^{2}$ is surrounded by a guard ring of approximately $8 \mathrm{~mm}$ width to isolate and drain leakage currents.

Taking the front side and the back side strips together, the detector can thus be divided into $128 \times 48$ pixels. The strips on the front side give the $x$-coordinate and the strips on the back side the $y$-coordinate. We note that these pixels are not square (see Fig. 2). This is intended as the detector was mainly designed for use with a crystal spectrometer. It requires a very good position resolution in the direction of the dispersion given by the $250 \mu \mathrm{m}$ wide strips, whereas the position resolution in the orthogonal direction is not as significant.

Each strip is read out separately with a charge sensitive preamplifier [type CSPA 02.04 Ref. 11)]. On the front side $\left(p^{+}\right.$contact), the positive charge carriers and, on the back side, the negative charge carriers are collected. The energy information is thus obtained for each strip on the front and the back side, respectively. For any true event the energy signal on both sides will be identical. This makes the detec- 


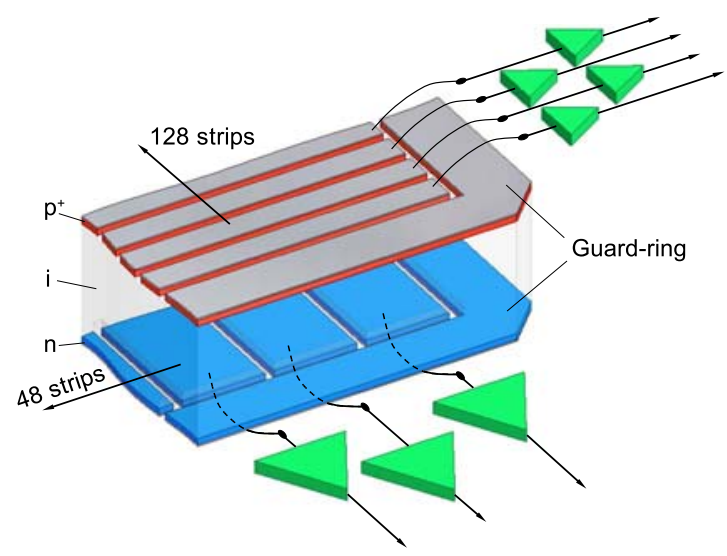

FIG. 1. (Color online) Schematic drawing of a small section of the Ge(i) crystal with etched orthogonal strips on front $(p)$ and back $(n)$ sides surrounded by a guard ring. Each strip is furnished with a separate readout.

tor truly multihit capable. In many multihit capable detector systems, for example, with delay lines, the correlation of the $x$-position with the correct $y$-position can be problematic for simultaneous hits. Using the energy information, however, the $x$ - and $y$-strips corresponding to a hit can be matched unambiguously. Even events where two hits fall on a single strip on either side can be reconstructed. This simultaneous detection of multiple hits is essential for a Compton polarimeter where the scattering event and the photoabsorption of the scattered photon occur in the same crystal.

In the measurements reported here, all 48 back side strips and 64 of the 128 front side strips have been separately connected to the readout electronics, enabling the coincident readout of the connected strips. The remaining 64 frontside strips were not read out. For the creation of the trigger signals, the back side strips have been connected to conventional main amplifiers with a short shaping time of $1 \mu \mathrm{s}$ and constant fraction discriminators. The timing signals have been linked by logical OR to a single master trigger signal. To obtain the energy spectra, all front and back side strips have been connected to highly integrated 16-fold spectroscopy amplifiers. All signals were digitized by VME based 32 channel analog-digital-convertors (ADCs). The data were then saved event by event in listmode format on tape and analyzed offline.

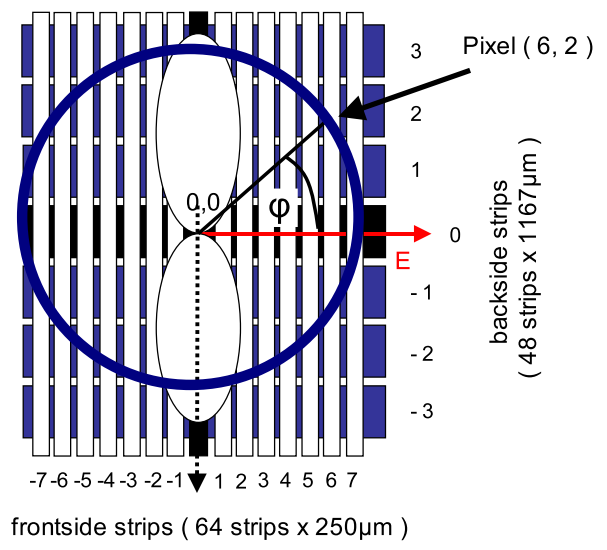

FIG. 2. (Color online) Numbering of the strips used in the text.

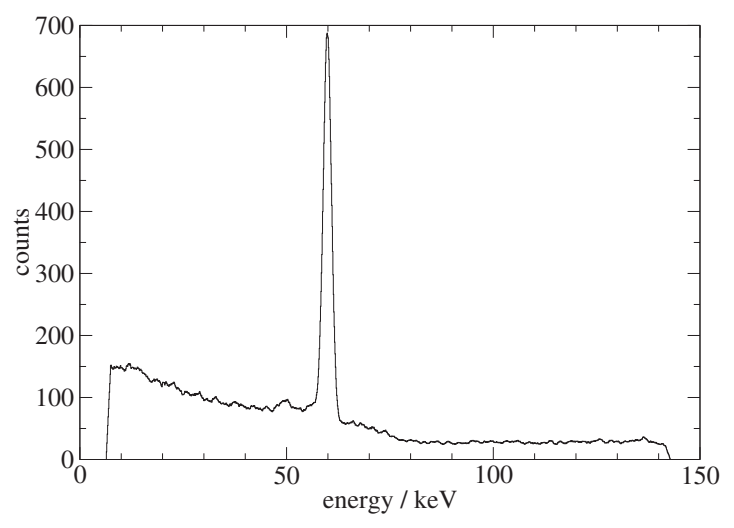

FIG. 3. Energy spectrum of a front side strip irradiated with $60 \mathrm{keV}$ photons.

\section{LINEARITY OF THE POSITION READOUT}

The plasma etching used to structure the detector crystal results in isolating grooves between the strips. To use the detector for imaging, a homogenous detection efficiency over the whole detector area must be ensured. Furthermore the influence of the isolating grooves on the detector response must be reproducible.

This influence has been investigated using $60 \mathrm{keV}$ photons at the beamline ID15A of the ESRF, which is optimized for photon energies in the range from $30 \mathrm{keV}$ up to several hundreds of $\mathrm{keV} .{ }^{12}$ The photon beam was focused to a square spot close to $50 \times 50 \mu \mathrm{m}^{2}$. A set of aluminum absorbers between the crystals of the monochromators was used to attenuate the high photon flux by several orders of magnitude to about $10^{3}$ photons $/ \mathrm{s}$.

The detector was mounted on a translation table, which could be precisely positioned with stepper motors. To protect the detector, a wall of lead bricks was built around the table. Before changing any beam parameters, the detector was moved behind this wall and the photon flux has been checked by a NaI detector. In addition the detector could be rotated around its axis. It was thus aligned with the $1167 \mu \mathrm{m}$ wide back side strips in a horizontal orientation (see Fig. 2).

The linearity of the position resolution was measured by moving the detector horizontally (perpendicular to the $250 \mu \mathrm{m}$ wide front side strips) in steps of $50 \mu \mathrm{m}$. 20 data points have been obtained with a measurement time of $600 \mathrm{~s}$ yielding between $1.5 \times 10^{5}$ and $2.3 \times 10^{5}$ events depending on the beam intensity.

The energy spectrum of the front strip hit by the beam is shown in Fig. 3. The photoabsorption of the $60 \mathrm{keV}$ photons dominates the spectrum. However, the peak is located on a broad background caused by different effects. The background above the photopeak and a certain percentage of the background below can be attributed to processes coming from the third harmonic (i.e., $180 \mathrm{keV}$ ), which is always present in the beam, but not visible in Fig. 3 due to the amplifier settings and limited ADC range. A certain part of the low energy background, however, can also be attributed to charge sharing between two neighboring strips. 


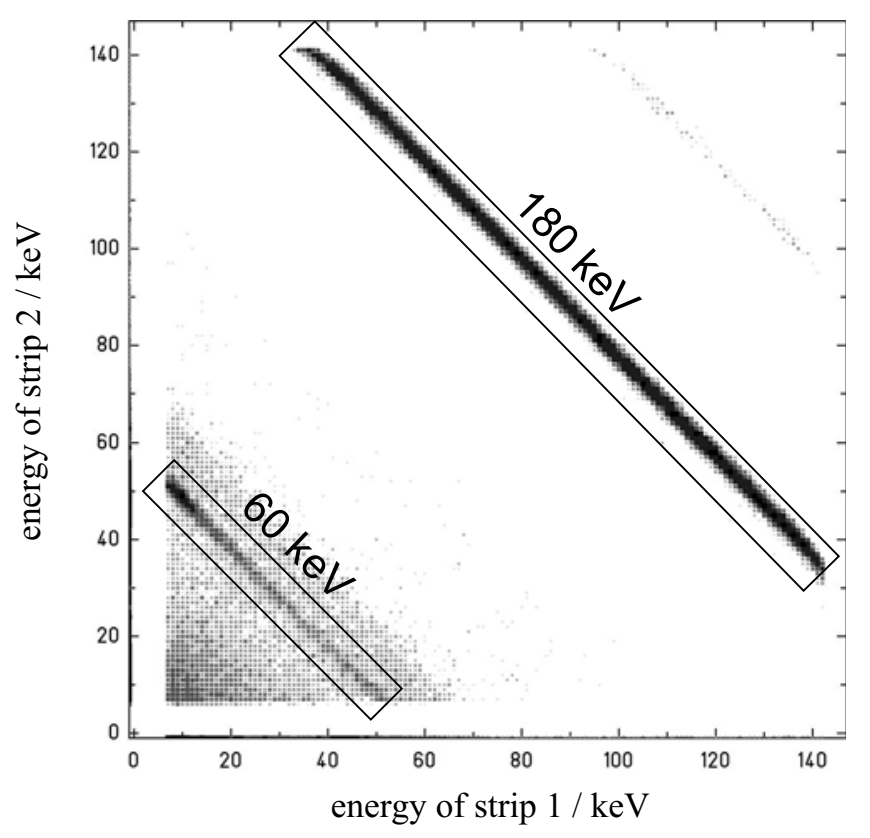

FIG. 4. Energy deposited on one strip as a function of the energy deposited in the neighboring strip in the same event. Charge sharing is visible as diagonals with constant sum.

\section{A. Charge sharing and position readout}

In the case of charge sharing, the sum energy of two neighboring strips will again yield the $60 \mathrm{keV}$ line. This is clearly demonstrated in Fig. 4, which shows the energy deposited on one strip versus the energy deposited in a neighboring strip. The two diagonal lines in the figure refer to charge sharing caused by photons with energies of 60 and $180 \mathrm{keV}$, respectively. The straight lines also show that there is no partial loss of charge in the grooves between the strips. The background of signals with a sum energy below $70 \mathrm{keV}$ can be attributed to Compton scattering, where the scattered photon escapes the detector and the deposited energy is distributed over two strips.

The charge sharing between strips is also reflected in the multiplicity of the event. The multiplicity gives the number of strips that have a signal above the noise. Figure 5 shows the measured multiplicity as a function of the beam position on the detector. With a period of $250 \mu \mathrm{m}$ (i.e., the strip

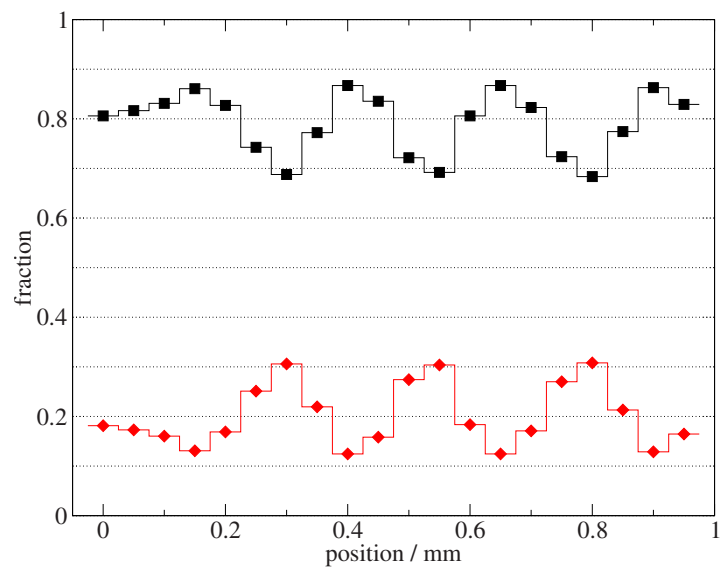

FIG. 5. (Color online) Fraction of multiplicity 1 (squares) and multiplicity 2 (diamonds) events as a function of the beam position on the detector.
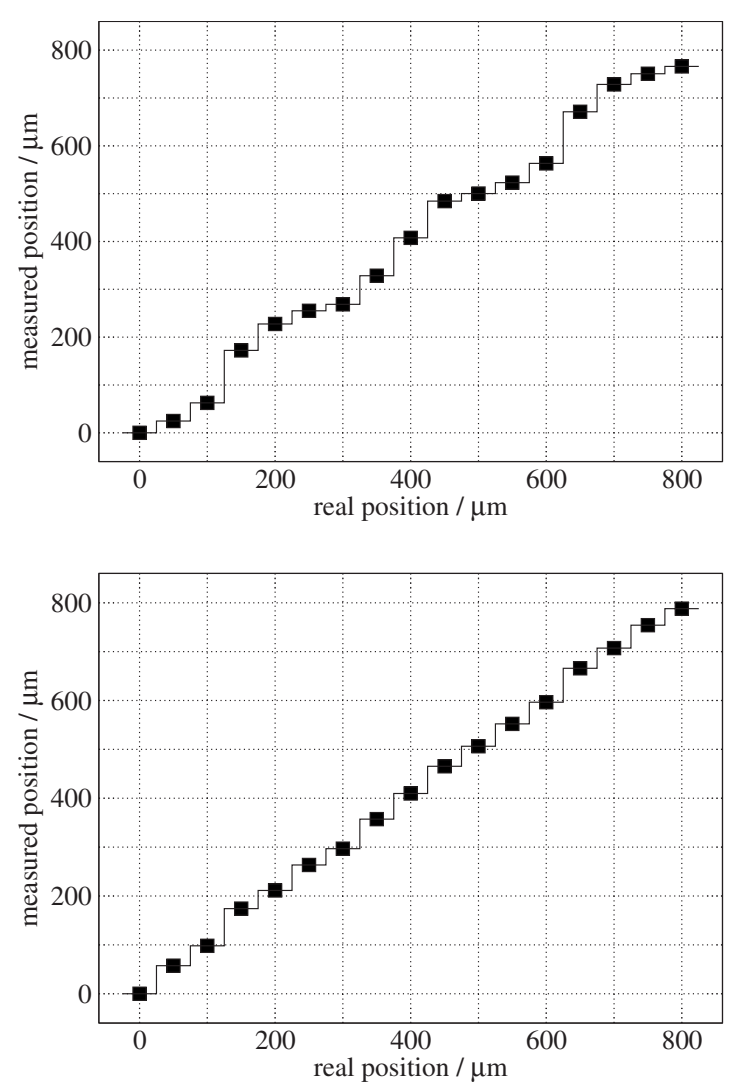

FIG. 6. Measured position on detector vs real position for multiplicity 1 events only (top) and multiplicity 1 and 2 events (bottom).

width) multiplicity " 1 " events show a minimum, and multiplicity "2" events a corresponding maximum. For these positions, the photon beam hits close to the edge of a strip or even between two strips. Then the sharing between the neighboring strips is expected to be maximal. Depending on the position, the contribution of multiplicity 2 events is between $15 \%$ and $30 \%$ and thus constitutes a significant amount of the detected events.

This charge sharing can be exploited to improve the position resolution of the detector. Figure 6 (top) shows the measured position as a function of the real position using only multiplicity 1 events. The influence of the strip width is clearly visible as discrete steps. For multiplicity 2 events resulting from charge sharing, a position between the strips may be assigned based on the fraction of charge deposited on each of the two strips. Considering all events, the beam position is determined by the center of the resulting distribution. A very good linearity between the measured position and the real position can thus be obtained, which is shown in Fig. 6 (bottom). As charge sharing should happen only between two neighboring strips, only multiplicity 2 events in neighboring strips have been used. This discards, for example, multiplicity 2 events from Compton scattering in the detector. The very good linearity clearly proves that a position resolution smaller than the strip width is possible, although not yet on an event by event basis.

\section{POLARIZATION MEASUREMENTS}

Typical Compton polarimeters consist of a scatterer and a detector to detect the Compton scattered photon. In the 


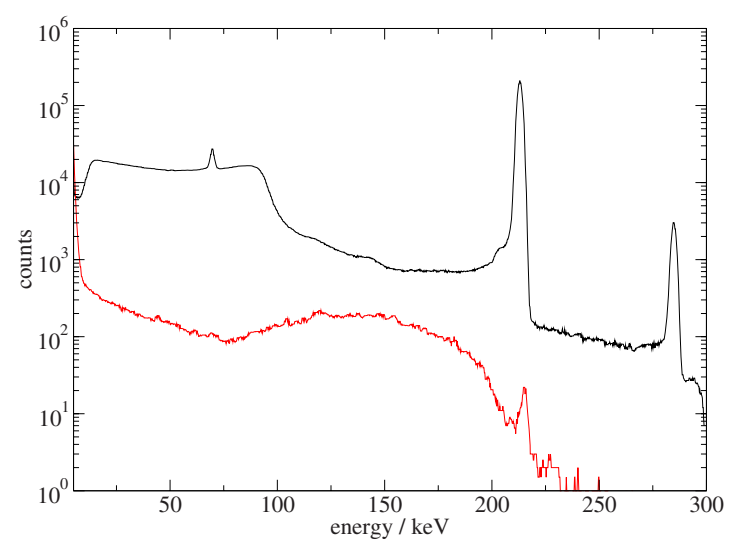

FIG. 7. (Color online) Energy spectra of the central back side strip (upper curve) and back side strip -5 (lower curve) without any conditions. The central strip is dominated by the photopeak and Compton distribution. The other strip is dominated by the broad distribution of the Compton scattered photons.

case of the detector presented here, the Ge(i) crystal acts simultaneously as scatterer and detector for the scattered photon.

\section{A. General considerations}

To investigate the detector response as a polarimeter, a beam of $98 \%$ linearly polarized photons with $210 \mathrm{keV}$ energy was focused to a spot size close to $50 \times 50 \mu \mathrm{m}^{2}$ on the detector. The beam was centered on one strip on the front and the back side, respectively. We will refer to these strips as strip 0 and number the other strips with respect to these two (see Fig. 2).

Figure 7 shows the energy spectrum of the central back side strip without any conditions. All x-rays regardless of their origin are shown. The spectrum is dominated by a strong line at $210 \mathrm{keV}$ coming from photoabsorption of photons from the third harmonic of the monochromator. From the linewidth the energy resolution is determined to be $\Delta E$ $=2.3 \mathrm{keV}$ full width at half maximum. A second photoabsorption peak lies at $70 \mathrm{keV}$ reflecting the first harmonic, while a third peak at $280 \mathrm{keV}$ is generated by pileup of the photons from both harmonics. The broad continuum below $100 \mathrm{keV}$ results from Compton scattered $210 \mathrm{keV}$ photons.

The energy $\Delta E$ deposited by the incident photon of energy $E$ during the Compton process and thus transferred to the Compton electron is given by

$$
\Delta E=E \frac{\frac{E}{m_{e} c^{2}}[1-\cos (\vartheta)]}{1+\frac{E}{m_{e} c^{2}}[1-\cos (\vartheta)]}
$$

and is a nonambiguous function of the Compton scattering angle $\vartheta$. For incident photon energies less than half the rest mass $m_{e} c^{2}$ of the electron, the energy of the scattered photon is always larger than the energy of the Compton electron (see Fig. 8). Thus the location of the scattering event and of the absorption of the scattered photon can be clearly identified. This also determined the choice of $210 \mathrm{keV}$ as the photon energy for the measurements presented here. It should be

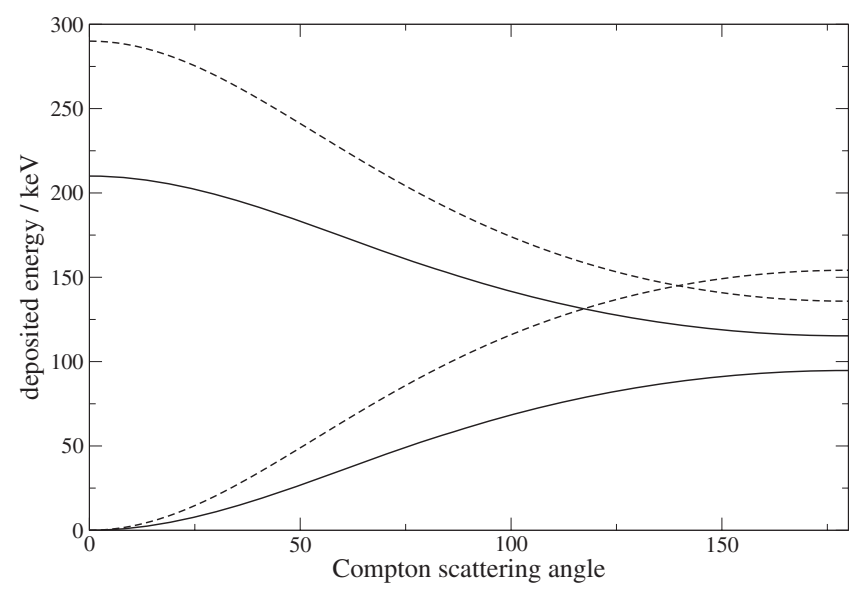

FIG. 8. Energy dependence of the Compton electron (lower curves) and scattered photon (upper curves) on the scattering angle $\vartheta$ for $210 \mathrm{keV}$ (solid line) and $290 \mathrm{keV}$ (dashed line) incident photon energies.

noted that for Compton polarimetry alone, this distinction is not needed due to the $\cos ^{2} \varphi$ dependence of the KleinNishina cross section (see below).

The probability that the photon will scatter by an angle $\vartheta$ is given by the Klein-Nishina differential cross section. In the case of $100 \%$ polarized light for a free electron at rest, this is given by

$$
\frac{d \sigma}{d \Omega}=\frac{r_{e}^{2}}{2}\left(\frac{E^{\prime}}{E}\right)^{2}\left(\frac{E^{\prime}}{E}+\frac{E}{E^{\prime}}-2 \sin ^{2} \vartheta \cos ^{2} \varphi\right),
$$

where $r_{e}$ is the classical electron radius and $E^{\prime}=E-\Delta E$ is the energy of the scattered photon. The azimuthal angle $\varphi$ describes the angle between the scattering plane and the polarization vector of the incident photon (see Fig. 9). It results in a typical dipole distribution with the largest scattering probability perpendicular to the polarization vector. The scattered photon with energy $E^{\prime}$ can also be absorbed in the crystal with a certain probability given by the detector geometry and the photoabsorption coefficient. The separate readout of the strips allows the coincident detection of the scattered photon. Only this allows the simultaneous use of the Ge(i) crystal as Compton scatterer and detector for the scattered photon. Its energy distribution is visible on the noncentral strips and also

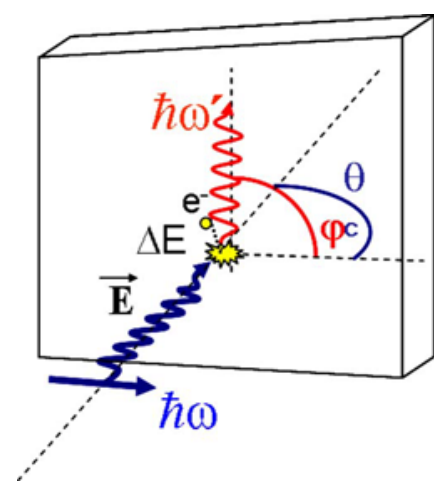

FIG. 9. (Color online) Compton scattering: A photon of energy $\hbar \omega$ is scattered at an electron under an angle of $\theta$ with respect to its original direction and transfers an energy of $\Delta E$ to the electron. The azimuthal angle $\varphi$ gives the direction of the scattered photon with respect to the polarization vector $\vec{E}$. 
shown in Fig. 7 for one back side strip as an example. It is complementary to the energy distribution of the Compton electrons.

\section{B. Reconstruction of Compton scattering events}

To determine the degree and orientation of the polarization of the incident photon, the complete Compton event in the detector must be reconstructed. This requires the determination of the position and amount of energy deposited by Compton scattering and subsequent photoabsorption of the scattered photon in the detector. All events where the incident photon undergoes multiple Compton scattering are discarded in our analysis. To reconstruct the complete Compton event from all signals, we apply the following algorithm on an event by event basis. First all pairs of front side and back side strips with the same deposited energy are searched for. Same energy in the context of the analysis presented here means an absolute energy difference of less than $5 \mathrm{keV}$ between the front side and the back side. This discards basically all strips that contain only noise or spurious signals. Each pair is then considered as a pixel of the twodimensional detector image. If exactly two pairs are found and the energy sum of both pairs is equal to the energy of the incident photon (i.e., $210 \mathrm{keV}$ ), we assume this to be a complete Compton event. This method will fail when the photon is scattered exactly in the direction of a strip. In this case the total energy will be deposited in this strip but is still distributed over two strips on the other side of the crystal. However, these events can still be reconstructed. If one side of the detector shows two strips with a signal above the noise, and the other side only one strip, we check whether the sum energy of the two strips equals the energy on the single strip and is equal to the energy of the incident photon. If this condition is fulfilled, the two pairs (i.e., pixels) can be calculated from the given data. All other cases, where only one or more than two pairs are found, are discarded in our analysis. With this algorithm about $7.5 \%$ of all initial Compton events in the detector can be reconstructed.

As the energy of the incident photon is less than the rest mass of the electron, the pixel with the lower energy value gives the position of the Compton scattering event and the pixel with the higher energy marks the location of the scattered photon's absorption. The first pixel marks the scattering center and will be designated as $(0,0)$ (see Fig. 2). Although the scattering center in the data presented here is fixed by the fact that the incident photon beam is centered on a single pixel, we do not use this information in our analysis. This makes our algorithm more generally applicable.

Figure 10 shows the position where the scattered photon is detected relative to the scattering center. The dipole shape due to the linear polarized incident photons is clearly visible. The curved right edge in the image coincides with the edge of the active area of the crystal. This can be attributed to a slightly reduced detector efficiency at these edges. The image also shows that some pixel in the center is missing. The central pixel contains the location of the Compton scatter event and is thus always empty in this display. The pixels immediately adjacent to the central pixel on the left/right and top/bottom have been discarded because of possible charge

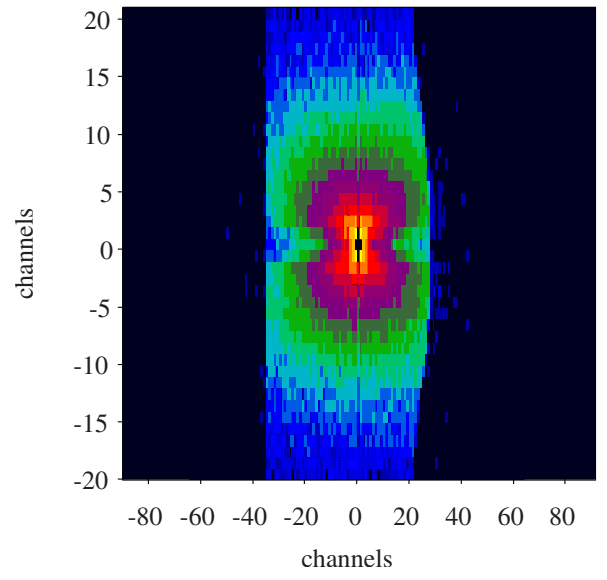

FIG. 10. (Color online) Position (in strips) of the scattered photon for all reconstructed complete Compton events.

sharing as discussed above. In these cases, we cannot distinguish between a complete Compton event taking place in two adjacent pixels or a photoabsorption event being distributed over two neighboring pixels by charge sharing. Therefore these inner pixels are discarded in our analysis. This does not constitute any loss of information as we will show below. As a final step in the analysis, the scattering angle $\vartheta$ is calculated from the energy of the Compton electron according to Eq. (1). The azimuthal angle $\phi$ with respect to the $x$-axis of the detector is derived from the position of the two pixels. The angle with respect to the polarization vector is then given by $\varphi=\phi-\phi_{0}$, where $\phi_{0}$ denotes the orientation of the polarization vector with respect to the $x$-axis.

\section{Compton scattering distributions}

The possibility to reconstruct the complete Compton event is not only determined by the respective cross sections for Compton and photoeffects. Geometrical effects such as the limited detector size or electronic side effects such as charge sharing between the strips and electronic noise play also an important role. This is in part due to the fact that the Ge(i) crystal acts simultaneously as Compton scatterer and absorber of the scattered photon. The probability to absorb the scattered photon thus strongly depends on the scattering angle $\vartheta$ due to the finite crystal thickness of $11 \mathrm{~mm}$. Photons scattered under forward or backward angles have a shorter path inside the crystal and thus a lower absorption probability than photons scattered under angles closer to $90^{\circ}$. This is presented in Fig. 11, which shows the energy distribution of the Compton scattered photons as a function of the distance from the scattering center. For a Compton event taking place in the center of the crystal at a depth of $5.5 \mathrm{~mm}$, the minimum and maximum scattering angles that can contribute at a distance $d$ from the center are given by $90^{\circ} \pm \tan ^{-1}(5.5 / d)$. For small distances $d$ this angular range is rather wide, resulting in a broad energy distribution of the scattered photon. For larger distances, the angular range centered around $90^{\circ}$ becomes narrower, which is reflected in a narrower energy distribution. Because we currently cannot measure the depth of the scattering event, our data are summed over the whole penetration depth washing out the above limits. Still, the 


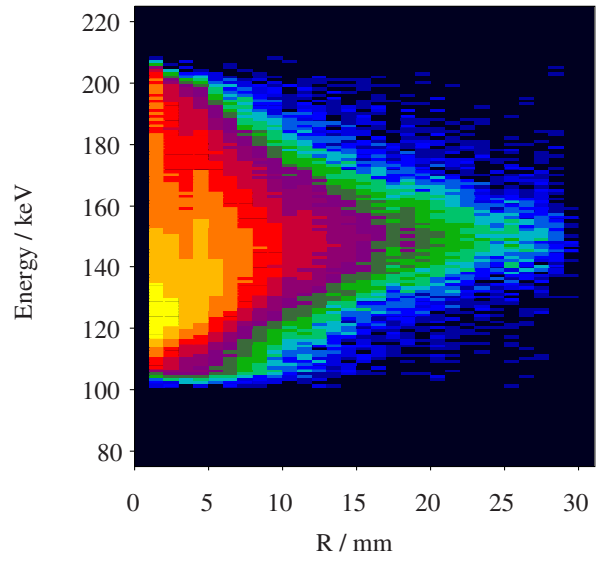

FIG. 11. (Color online) Energy distribution of the absorbed Compton scattered photon as a function of the distance from the scattering center.

geometrical effect is large enough to be clearly visible in Fig. 11. The obvious asymmetry of the energy distribution at small distances can be attributed to the energy dependence of the photoabsorption coefficient, i.e., the cross section is smaller for scattering into the forward direction (higher photon energies) as compared to those scattered in the backward direction.

The detector geometry along with the differential Compton scattering cross section results in a strong reduction of the efficiency for the detection of Compton events for scattering into the forward and backward hemispheres. This is most obvious in Fig. 12, which shows the energy distribution of the Compton electrons and scattered photons for all reconstructed complete Compton events.

By comparing the respective distributions in Figs. 7 and 12 , it is evident that the highest efficiency to reconstruct the complete Compton event is obtained for scattering angles close to $\vartheta=90^{\circ}$ where the differential Compton cross section is most sensitive to the polarization [see Eq. (2)]. This is shown in Fig. 13, which displays the position of the Compton photons scattered into a cone with opening angle $\Delta \vartheta$ $= \pm 10^{\circ}$ and scattering angles (from left to right) $\vartheta=30^{\circ}$, $50^{\circ}, 70^{\circ}, 90^{\circ}, 110^{\circ}, 130^{\circ}, 150^{\circ}, 170^{\circ}$. The distributions displayed give a direct visualization of the $\vartheta$ and $\varphi$ depen-

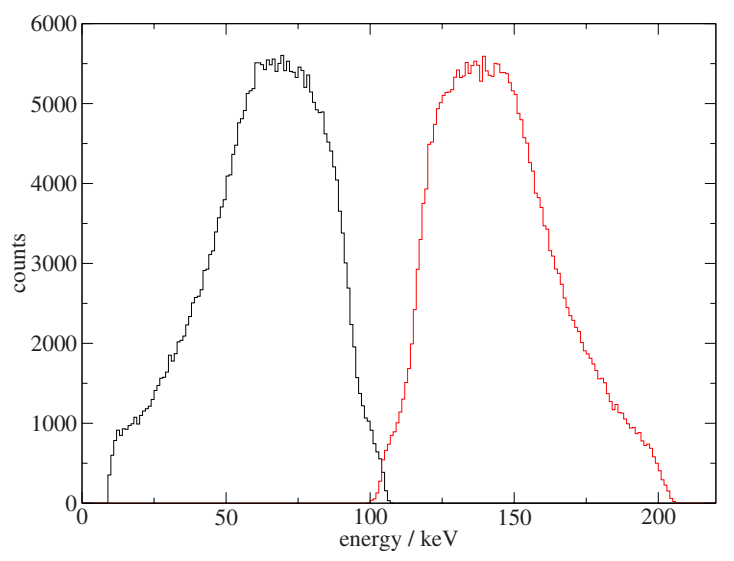

FIG. 12. (Color online) Energy distribution of the Compton electrons (left) and scattered photons (right) for complete Compton events detected.

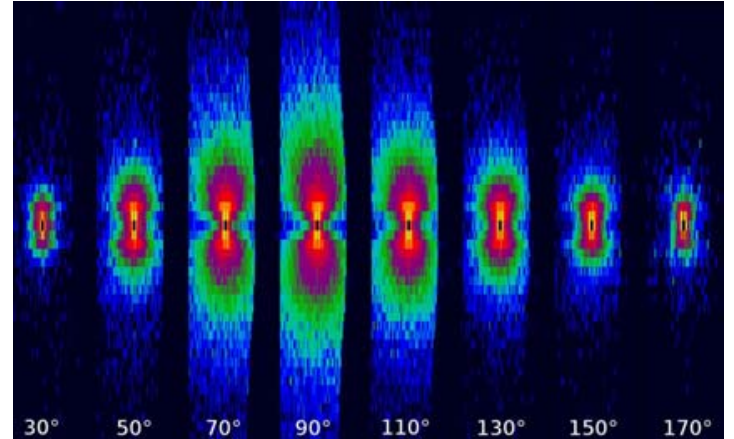

FIG. 13. (Color online) Position of the photons scattered into a cone with opening angle $\Delta \vartheta= \pm 10^{\circ}$ and scattering angles (from left to right) $\vartheta=30^{\circ}$, $50^{\circ}, 70^{\circ}, 90^{\circ}, 110^{\circ}, 130^{\circ}, 150^{\circ}, 170^{\circ}$ for all reconstructed complete Compton events.

dences of the Klein-Nishina cross section for linearly polarized photons [see Eq. (2)].

\section{Consistency check: Determination of the linear absorption coefficient}

To further analyze the sensitivity of the detector to the polarization of the incident photons, we now consider only events with a scattering angle $\vartheta=90^{\circ} \pm 10^{\circ}$. Besides the advantages discussed above, the Klein-Nishina cross section is most insensitive to variations in $\vartheta$ for this angle due to the $\sin ^{2} \vartheta$ dependence. The range of $\Delta \vartheta= \pm 10^{\circ}$ is a compromise between the required statistics and the influence of the geometrical effects discussed above.

The information about the polarization is obtained by analyzing the $\phi$-distribution of the complete Compton events. The fact that the active detector area is not square has to be taken into account as well as the effect of the finite pixel size. The influence of the finite pixel size will be most pronounced for small distances from the scattering center. As an example we present in Fig. 14 the $\phi$-distribution for distances between 3-4 and 6-7 mm from the center. The number of points used in Fig. 14 has been chosen so that the angle covered by one point is in the same order as the maximum angle covered by one pixel as seen from the center. This is to reduce the effects of the finite pixel size. Also

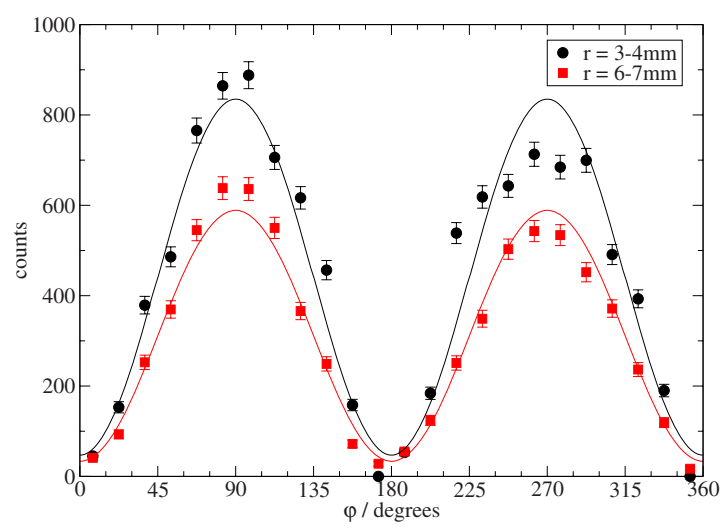

FIG. 14. (Color online) Dependence of the complete Compton events on the azimuthal angle $\phi$ for distances between 3-4 $\mathrm{mm}$ (circles) and 6-7 $\mathrm{mm}$ (squares). The solid lines represent a fit of the Klein-Nishina cross section with $\vartheta=90^{\circ}$. 


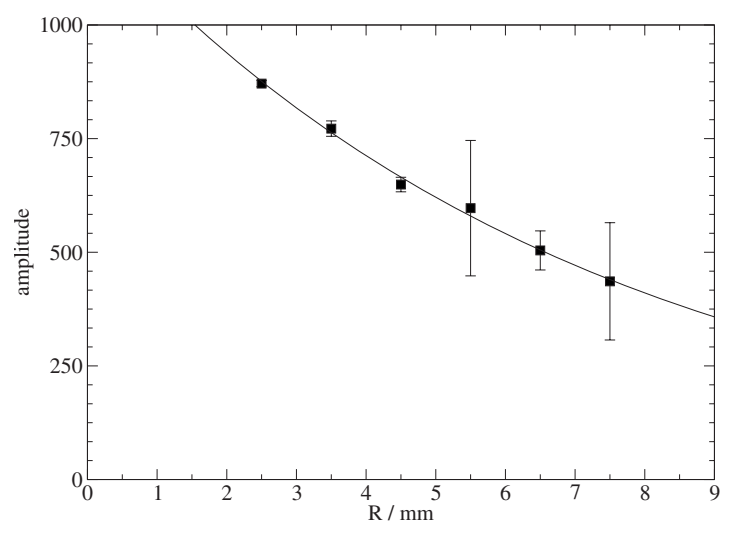

FIG. 15. Dependence of the fitted proportionality factor from the KleinNishina fit (see Fig. 14 and text) on the distance from the center. The solid line is a fitted exponential decay curve.

shown in Fig. 14 is a fit of the Klein-Nishina cross section [Eq. (2)] with the amplitude as the only fit parameter. The experimental data for both distances are very well reproduced by this fit. The slightly different heights of the two maxima at $\phi=90^{\circ}$ and $\phi=270^{\circ}$ can be attributed to the possibility that the photon beam did not hit the exact center of the central pixel. This is supported by the fact that the difference in height becomes less pronounced for larger distances from the center.

The dependence of the fitted amplitude on the distance from the center is shown in Fig. 15 and can be used as a cross check on the influence of geometrical effects on the efficiency. In the absence of such effects, an exponential decay fit to the distance dependence must yield the total linear absorption coefficient $\mu$ for the scattered photons (photoabsorption plus Compton scattering). A fit to the experimental data (see Fig. 15) gives $\mu=0.138 \pm 0.005 \mathrm{~mm}^{-1}$. This is in very good agreement with the NIST value of $\mu=0.135 \mathrm{~mm}^{-1}$ (Ref. 13) for the $148.9 \mathrm{keV}$ photons after scattering under $\vartheta=90^{\circ}$.

\section{E. Polarization sensitivity}

To assess the relative effectiveness of any arrangement of detectors as a polarimeter, the response to a $100 \%$ polarized beam of photons is used, either calculated or measured. Given the 98\% linear polarization of the incident photons, we can directly extract the response of the detector from the data presented above. This response is known as the polarimetric modulation factor $Q$ and is given by Suffert et al. ${ }^{14}$ as

$$
Q=\frac{N_{\perp}-N_{\|}}{N_{\perp}+N_{\|}}
$$

where $N_{\perp}$ and $N_{\|}$are the detected Compton events with scattering perpendicular and parallel to the polarization vector. The theoretical value of $Q$ for an ideal detector can be obtained from the Klein-Nishina cross section and is given by

$$
Q=\frac{\sin ^{2} \vartheta}{\epsilon^{-1}+\epsilon-\sin ^{2} \vartheta},
$$

where $\epsilon=E^{\prime}$ / $E$ is the ratio between the scattered photon energy $E^{\prime}$ and the incident photon energy $E$. The modulation factor and thus the sensitivity is maximal for a scattering

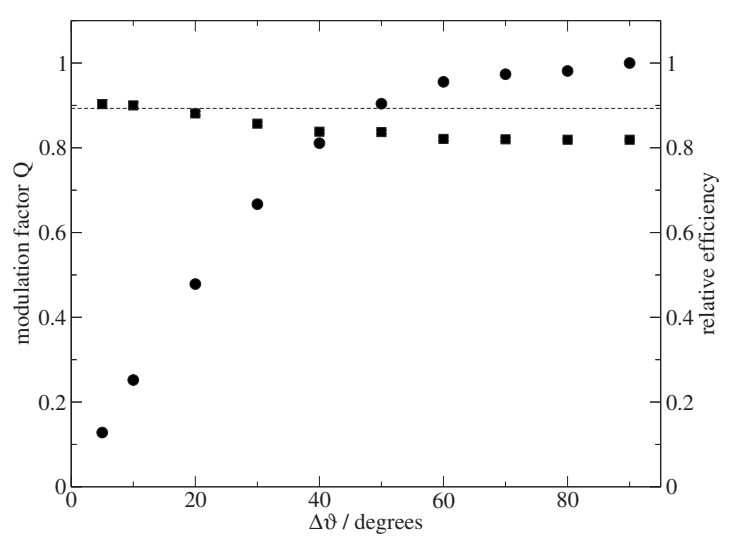

FIG. 16. Dependence of the modulation factor $Q$ (squares) and the relative efficiency (circles) on the opening angle $\Delta \vartheta=90^{\circ}$. The dashed line indicates the theoretical modulation factor $Q=0.893$ for an ideal detector.

angle of $\vartheta=90^{\circ}$ and decreases for angles toward $\vartheta=0^{\circ}$ and $\vartheta=180^{\circ}$. For $E=210 \mathrm{keV}$ the maximum sensitivity is $Q=0.894$.

Our analysis as described above is similar to the radial bin technique and decoupled ring technique described by Lei et al. ${ }^{1}$ To obtain the modulation factor from the measured $\phi$-distribution we write Eq. (2) for $\vartheta=90^{\circ}$ as

$$
\frac{d \sigma}{d \Omega}(\phi)=A-B \cos 2\left(\phi-\phi_{0}\right),
$$

where we have used the relation $\cos ^{2} x=(1+\cos 2 x) / 2$. The modulation factor $Q$ can be obtained by fitting the $\phi$-distribution for $\vartheta=90^{\circ}$ with this function. It is then given by

$$
Q=\frac{B}{A} .
$$

Following the above discussion we now consider all complete Compton events within a distance of 2-8 $\mathrm{mm}$ from the center and fit the experimental $\phi$-distribution with Eq. (5). We obtain for the modulation factor $Q=0.900 \pm 0.007$ and for the polarization angle $\phi_{0}=0.3^{\circ} \pm 0.3^{\circ}$. The measured modulation factor of the detector is thus in very good agreement with the theoretical value for an ideal detector. This makes two-dimensional microstrip detectors like the one presented here ideal for polarization measurements.

To improve the statistics in low count rate experiments, it may be necessary to use a broader range $\Delta \vartheta$ and accept a deterioration of the modulation factor $Q$. Figure 16 shows the decrease of the $Q$ factor with increasing opening angle $\Delta \vartheta$. For $\Delta \vartheta=90^{\circ}$ all complete Compton events detected are considered. The modulation factor decreases with increasing opening angle as expected but reaches a saturation of 0.82 . This is due to the finite thickness of the detector, which already preselects Compton events with scattering angle around $\vartheta=90^{\circ}$. The figure also shows the relative efficiency normalized to the case $\Delta \vartheta=90^{\circ}$. Here a significant increase in efficiency can be obtained by considering all complete Compton events within $\vartheta=90^{\circ} \pm 40^{\circ}$ with a modulation factor of about 0.84 . 


\section{COMPTON SCATTERING FROM ATOMS}

The analysis of the polarization sensitivity discussed above is based on the Klein-Nishina formula for a free electron. As Compton scattering in the detector involves bound electrons, the influence of the initial electron momentum must in principle be taken into account. We neglected this in the above discussion for the following reasons. The energy transferred to the electron is typically $70 \mathrm{keV}$ (see Fig. 12), which corresponds to a momentum transfer of $k=18.8$ a.u. In germanium with a nuclear charge $Z=32$, only the $K$-shell electrons have a larger average momentum of $a=28.6$ a.u. The probability of Compton scattering on a $K$-shell electron in germanium is only about $6 \%$ and can thus be neglected. For all other shells, the condition $a / k<1$ is fulfilled.

While the results presented above support this simplification, the initial momentum of the bound electron may become significant at lower photon energies. For the description of Compton scattering from atoms, the impulse approximation is commonly used. This involves the assumption that scattering from the bound electrons can be described as scattering from a free electron with a momentum distribution of the bound electron, i.e., its Compton profile. Simulations of the detector response using Monte Carlo codes such as the EGS5 code system ${ }^{15}$ can include the impulse approximation. However, the region of validity of the impulse approximation is still under discussion. ${ }^{16,17}$ Future Compton polarimeters with higher resolution as the one presented here may even be used to test the validity of the impulse approximation.

\section{CONCLUSION AND OUTLOOK}

We have demonstrated the superior capabilities of a twodimensional microstrip Ge(i) detector for imaging and as a Compton polarimeter. While it can easily perform like an ideal detector, a good compromise between efficiency and sensitivity can be achieved with the polarimeter modulation factor $Q$ still being within $94 \%$ of the theoretical value. As the current detector was not designed for Compton polarimetry, it suffers from nonsquare pixels and a rather small size in the horizontal direction. A future two-dimensional microstrip detector dedicated to Compton polarimetry will have a large area of about $64 \times 64 \mathrm{~mm}^{2}$ with 32 strips each on the front and back sides. This will result in square pixels of $2 \times 2 \mathrm{~mm}^{2}$ size. Furthermore, to increase the efficiency for Compton scattering of incident photons below $100 \mathrm{keV}$ energy, Li drifted Si will be used instead of high purity Ge.

\section{ACKNOWLEDGMENTS}

We acknowledge the European Synchrotron Radiation Facility for provision of synchrotron radiation facilities and we would like to thank the staff and especially Dr. Th. d'Almeida for the excellent support in using beamline ID15A. We thank S. Tashenov, D. Banas, J. Chatterjey, C. Brandau, Ch. Kozhuharov, and N. Kurz for their support in preparation of and during the beamtime. We also thank M. Kavcic and J. Szlachetko for their participation in the beamtime at the ESRF.

${ }^{1}$ F. Lei, J. Dean, and G. Hills, Space Sci. Rev. 82, 309 (1997).

${ }^{2}$ T. Stöhlker, D. Banas, H. Beyer, A. Gumberidze, S. Tachenov, D. Sierpowski, T. Krings, C. Kozhuharov, U. Spillmann, X. Ma, and D. Protic, Nuclear Science Symposium Conference Record, 2002 (IEEE, New York, 2002), Vol. 1, p. 366.

${ }^{3}$ T. Stöhlker, D. Banas, H. Beyer, A. Gumberidze, C. Kozhuharov, E. Kanter, T. Krings, W. Lewoczko, X. Ma, D. Protic, D. Sierpowski, U. Spillmann, S. Tachenov, and A. Warczak, Nucl. Instrum. Methods Phys. Res. B 205, 210 (2003).

${ }_{5}^{4}$ J. Eichler and T. Stöhlker, Phys. Rep. 439, 1 (2007).

${ }^{5}$ H. Beyer, T. Stöhlker, D. Banas, D. Liesen, D. Protic, K. Beckert, P. Beller, J. Bojowald, F. Bosch, and E. Förster, Spectrochim. Acta, Part B 59, 15351542 (2004).

${ }^{6}$ S. Tashenov, T. Stöhlker, D. Banas, K. Beckert, P. Beller, H. Beyer, F. Bosch, S. Fritzsche, A. Gumberidze, S. Hagmann, C. Kozhuharov, T. Krings, D. Liesen, F. Nolden, D. Protic, D. Sierpowski, U. Spillmann, M. Steck, and A. Surzhykov, Phys. Rev. Lett. 97, 223202 (2006).

${ }^{7}$ T. Stöhlker, H. Beyer, H. Bräuning, A. Bräuning-Demian, C. Brandau, S. Hagmann, C. Kozhuharov, H. Kluge, T. Kühl, D. Liesen, R. Mann, W. Nörtershäuser, W. Quint, U. Schramm, and R. Schuch, Nucl. Instrum. Methods Phys. Res. B 261, 234 (2007).

${ }^{8}$ International accelerator facility for beams of ions and antiprotons, 2001 (URL http://www.gsi.de/GSIFuture/cdr/).

${ }^{9}$ D. Protic, T. Stöhlker, T. Krings, I. Mohos, and U. Spillmann, IEEE Trans. Nucl. Sci. 52, 3194 (2005).

${ }^{10}$ S. Chatterjee, H. Beyer, D. Liesen, T. Stöhlker, A. Gumberidze, C. Kozhuharov, D. Banas, D. Protic, K. Beckert, P. Beller, T. Krings, F. Bosch, B. Franzke, S. Hagmann, J. Hoszowska, P. Indelicato, H.-J. Kluge, X. Ma, B. Manil, I. Mohos, F. Nolden, U. Popp, A. Simionovici, D. Sierpowski, M. Steck, U. Spillmann, C. Brandau, E. Forster, Z. Stachura, S. Tashenov, M. Trassinelli, A. Warczak, O. Werhan, E. Ziegler, S. Trotsenko, and R. Reuschl, Nucl. Instrum. Methods Phys. Res. B 245, 67 (2006).

${ }^{11}$ CSPA 02.04, KFKI-Budapest, Hungary.

${ }^{12}$ P. Suortti and T. Tschentscher, Rev. Sci. Instrum. 66, 1798 (1995).

${ }^{13}$ C. Chantler, K. Olsen, R. Dragoset, J. Chang, A. Kishore, S. Kotochigova, and D. Zucker, X-ray Form Factor, Attenuation and Scattering Tables (Version 2.1) (National Institute of Standards and Technology, Gaithersburg, MD, 2005); originally published as C. T. Chantler, J. Phys. Chem. Ref. Data 29, 597 (2000); 24, 71 (1995).

${ }^{14}$ M. Suffert, P. Endt, and A. Hoogenboom, Physica (Amsterdam) 25, 659 (1959).

${ }^{15}$ H. Hirayama, Y. Namito, A. Bielajew, S. Wilderman, and W. Nelson, Technical Report No. SLAC-R-730, 2005.

${ }^{16}$ R. Pratt, L. LaJohn, T. Suric, B. Chatterjee, and S. Roy, Nucl. Instrum. Methods Phys. Res. B 261, 175 (2007).

${ }^{17}$ Z. Kaliman, T. Suric, K. Pisk, and R. Pratt, Phys. Rev. A 57, 2683 (1998). 